\title{
A PLANT COLLECTION FROM THE COCHRANE RIVER REGION OF NORTHWESTERN MANITOBA
}

\author{
by George W. Scotter ${ }^{1}$
}

During the summer field season of 1963, the Canadian Wildlife Service continued a study on the effects of forest fires on the winter range of Barren-ground Caribou (Rangifer tarandus groenlandicus). The Cochrane River region of northwestern Manitaba was chosen as the study site. Incidental to the major study, the writer made collections of vascular plants, bryophytes, and lichens. The purpose of this paper is to record the vascular plants collected in this rather poorly known area.

The study site extended from $101^{\circ}$ W. longitude to the SaskatchewanManitoba boundary and from $57^{\circ} 30^{\prime}$ $N$. latitude to the District of Keewatin-Manitoba boundary. The collection sites were situated within small radii of the following locations:

$\begin{array}{ll}\text { Brochet ........... } 57^{\circ} 54^{\prime} & 101^{\circ} 40^{\prime} \\ \text { Cochrane River.. } 58^{\circ} 25^{\prime} & 101^{\circ} 10^{\prime} \\ \text { Fort Hall Lake.. } 59^{\circ} 16^{\prime} & 101^{\circ} 27^{\prime} \\ \text { Horseshoe Lake. } 57^{\circ} 43^{\prime} & 101^{\circ} 15^{\prime} \\ \text { Kasmere Lake .. } 59^{\circ} 34^{\prime} & 101^{\circ} \mathbf{1 0}^{\prime} \\ \text { Lac Brochet ..... } 58^{\circ} 38^{\prime} & 101^{\circ} 40^{\prime} \\ \text { Thanout Lake .... } 59^{\circ} 23^{\prime} & 101^{\circ} 20^{\prime} \\ \text { Whiskey Jack } & \\ \text { Lake ........... } 58^{\circ} 25^{\prime} & 101^{\circ} 55^{\prime}\end{array}$

Although the present list of vascular plants adds no species to the Flora of Manitoba (Scoggan, 1957), several of the collections represent extensions of previously known ranges within the province. These include Woodsia alpina, Triglochin maritima, Carex houghtonii, Juncus arcticus, Luzula wahlenbergii, Orchis rotundifolia, Salix scouleriana, Erucastrum gallicum, Arabis drummondii, Pedicularis labradorica, Petasites sagittatus, and Arnica alpina subsp. attenuata which were not recorded from the Reindeer-Nueltin lakes area of northwestern Manitoba by Baldwin (1953). Other contributions to the flora of northern Manitoba have been summarized by Scoggan (1957). Since that date Ritchie $(1959,1962)$ has added to the knowledge of the flora of the northern region.

For the most part, the nomenclature used in the species list is that of Scoggan (1957). Data on collection habitat, locality, and the writer's col- lection number are included. All specimens cited have been deposited in the National Herbarium of Canada. Duplicate material, where available, has been deposited in the herbaria of the Canadian Wildife Service at Edmonton, Alberta and the Department of Botany and Plant Pathology at Logan, Utah. Dr. H. J. Scoggan, National Herbarium of Canada, identified or verified identification of all the collection. Dr. G. W. Argus, of the W. P. Fraser Herbarium at Saskatoon, later revised part of the Salix collection.

\section{SPECIES LIST}

Equisetum arvense L. Moist site in mature white birch forest. Fort Hall L. 3781 .

Equisetum arvense var. boreale (Bong.) Klinge Sandy beach. Fort Hall L. 3724.

Equisetum scirpoides Michx. Muskeg site. Kasmere L. 3660.

Lycopodium clavatum var. monostachyon Grev. \& Hook. On an esker. Kasmere L. 3663.

Lycopodium complanatum L. Mature black spruce-jack pine forest. Cochrane $R .3674$.

Lycopodium obscurum L. Mature black spruce-jack pine forest. Cochrane $R .3675$.

Lycopodium annotinum var. pungens (LaPylaie) Desv. Mature black spruce-jack pine forest. Cochrane $\mathbf{R}$. 3675 .

Dryopteris robertiana (Hoffm.) Christens. Rocky cliff. Fort Hall L. 3728.

Dryopteris fragrans (L.) Schott Rocky cliff. Fort Hall L. 3729.

Cystopteris fragilis (L.) Bernh. Rocky ledge. Fort Hall L. 3725.

Woodsia alpina (Bolton) S. F. Gray Rocky ledge. Fort Hall L. 3726, 3730.

Triglochin maritima L. Growing in shallow water. Kasmere L. 3650.

Festuca saximonteana Rydb. Sandy beach near Indian campsite. Lac Brochet 3718 .

\footnotetext{
${ }^{1}$ Range Ecologist, Canadian Wildlife Service, 742 Federal Building, Edmonton, Alberta.
} 
Poa alpigena (Fr.) Lindm. Sandy area. Horseshoe L. 3748.

Poa palustris L. Sandy areas and disturbed areas. Horseshoe L. 3745; Lac Brochet 3692, 3693, 3694, 3719.

Poa glauca Vahl Sandy shore. Kasmere L. 3647.

Trisetum spicatum (L.) Richter Opening near old trading post. Thanout L. 3712 .

Agrotis scabra Willd. Sandy area. Horseshoe L. 3749.

Calamagrostis purpurascens $\mathrm{R} . \mathrm{Br}$. Sandy shore. Kasmere L. 3648.

Calamagrostis canadensis (Michx.) Nutt. Sandy shore. Kasmere L. 3737.

Eriophorum angustifolium Honckeny Muskeg sites. Kasmere L. 3651; Fort Hall L. 3722 .

Eriophorum spissum Fern. Muskeg. sites. Kasmere L. 3652; Cochrane R. $3690,3714$.

Carex foenea Willd. (C. siccata Dew.) Sandy esker. Kasmere L. 3645.

Carex disperma Dew. Moist mature birch forest. Kasmere L. 3641.

Carex brunnescens (Pers.) Poir. Muskeg site. Fort Hall L. 3733.

Carex aenea Fern. Recently burntover site. Whiskey Jack L. 3736.

Carex deflexa Hornem. Sandy shore. Cochrane R. 3691.

Carex glacialis Mack. Sandy esker. Kasmere L. 3638.

Carex houghtonii Torr. Sandy lake shore. Horseshoe L. 3739.

Carex paupercula Michx. Muskeg site. Fort Hall L. 3723.

Carex aquatilis Wahl. Sandy beach near lake. Fort Hall L. 3720; Kasmere L. 3640 .

Carex saxatilis L. var. miliaris (Michx.) Bailey On lake shore. Kasmere L. 3639 .

Carex rotundata Wahl. Lake shore. Kasmere L. 3642.

Juncus arcticus Willd. Sandy shore. Font Hall L. 3732.

Luzula wahlenbergii Rupr. Sandy shore. Kasmere L. 3665.

Tofieldia pusilla ('Michx.) Pers. Muskeg site. Kasmere L. 3662.

Smilacina trifolia (L.) Desf. Muskeg site. Kasmere L. 3655.

Orchis rotundifolia Banks Moist site in an open black spruce-forest.
Thanout L. 3684.

Salix glauca L. Muskeg site. Kasmere L. 3657.

Salix myrtillifolia Anderss. Sandy hillside and boggy sites. Kasmere L. $3629,3630,3658$.

Salix pyrifolia Anderss. Burnt-over area. Lac Brochet 3698.

Salix bebbiana Sarg. On sandy shore and sandy esker. Lac Brochet 3672 ; Fort Hall L. 3734.

Salix scoulerianci Barratt Recently burnt-over area. Lac Brochet 3701.

Alnus rugosa (Du Roi) Spreng. var. americana (Regel) Fern. Lake shore. Horseshoe L. 3746.

Betula pumila L. var. glandulifera Regel Kasmere L. 3686, 3687.

Betula papyrifera var. humilis (Regel) Fern. \& Raup. Horseshoe L. $3740,3741,3743,3744$; Kasmere L. $3631,3633,3635,3636$; Cochrane R. 3677.

Stellaria ciliatosepala Trautv. Sandy shore. Fort Hall L. 3709.

Stellaria longifolia Muhl. Near lake shore. Lac Brochet 3704.

Ranunculus lapponicus L. Along a stream bank and in a mature black spruce muskeg. Kasmere L. 3649; Lac Brochet 3670 .

Corydalis sempervirens ( $\left.\mathrm{I}_{\text {. }}\right)$ Pers. Recently burnt-over area. Lac Brochet 3699.

Draba nemorosa L. var. lejocarpa Lindbl. Recently burnt-over area. Lac Brochet 3695 .

Erucastrum gallicum (Willd.) O. E. Schulz Solitary specimen growing near lake shore. Thanout L. 3711.

Barbarea orthoceras Ledeb. Sandy shore. Lac Brochet 3673.

Cardamine pensylvanica Muhl. Solitary specimen on sandy shore. Lac Brochet 3717.

Arabis drummondii Gray. Sandy esker. Kasmere L. 3644.

Saxifraga tricuspidata Rottb. Along an esker. Kasmere L. 3646.

Ribes glandulosum Grauer Sandy sites. Kasmere L. 3666; Horseshoe L. 3747.

Potentilla norvegica L. Clearing near abandoned Fort Hall. Thanout L. 3710 .

Rubus chamaemorus L. Muskeg 


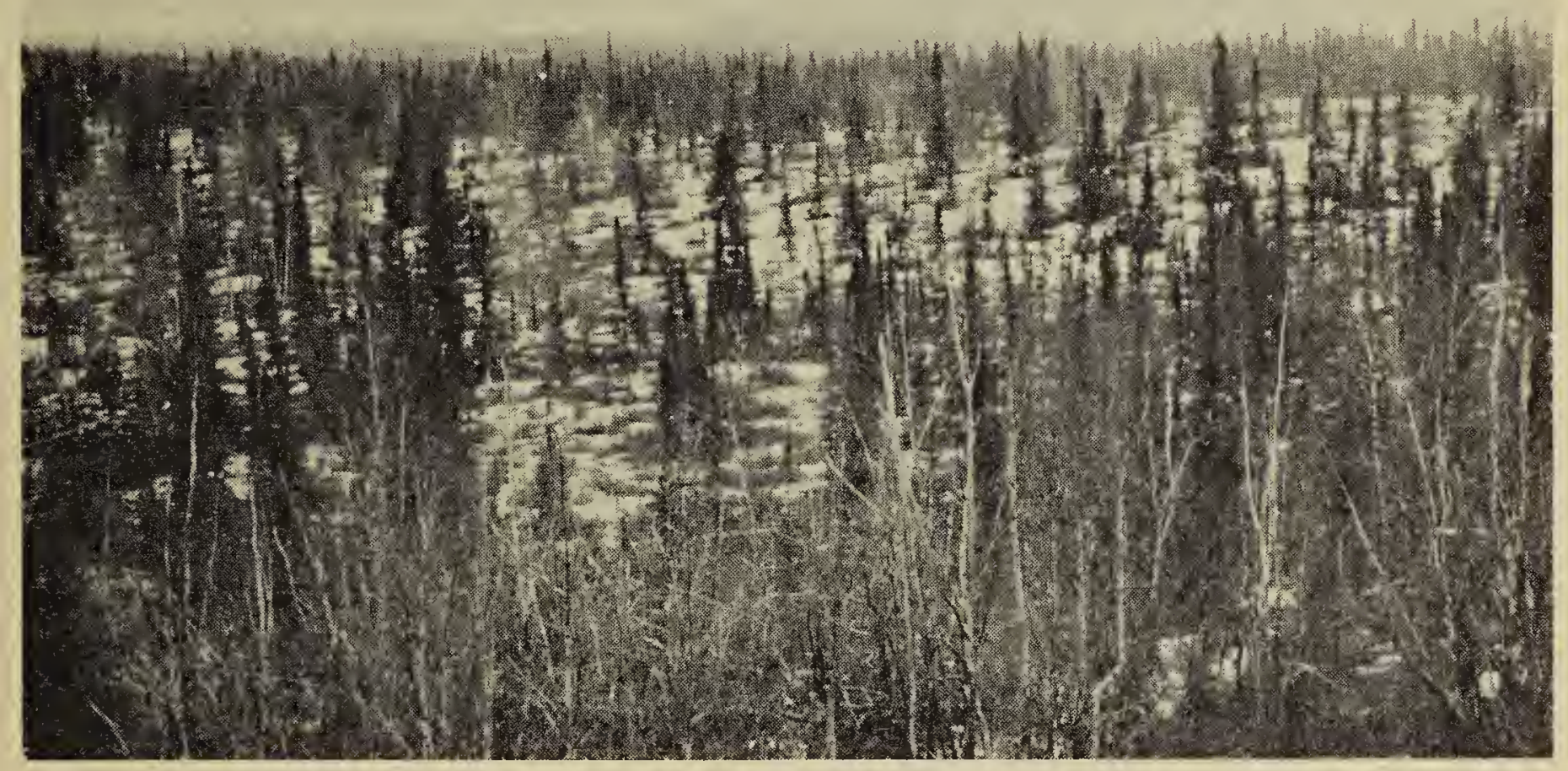

Photo by Ermio Kuyt

Open spruce forest, characteristic of the region

sites. Kasmere L. 3706; Cochrane.R. 3702.

Rubus acaulis Michx. Moist, mature birch forest. Kasmere L. 3643.

Prunus pensylvanica L.f. Sandy shore. Cochrane R. 3716.

Epilobium palustre var. oliganthum (Michx.) Fern. Recently burnt-over muskeg site. Fort Hall L. 3683.

Cicuta mackenzieana Raup Rocky lake shore. Brochet 3738 .

Cornus canadensis L. Opening near old cabins. Lac Brochet 3696.

Pyrola grandiflora Radius Shaded site on rocky hillside. Fort Hall L. 3727.

Ledum groenlandicum Oeder Moist, recently burnt-over site. Lac Brochet 3700.

Ledum palustre L. var. decumbens Ait. Dry muskeg site. Kasmere L. 3664 .

Kalmia polifolia Wang. Muskeg site. Cochrane R. 3715.
Chamaedaphne calyculata (L.) Moench Moist, rocky shore line. Cochrane R. 3688 .

Andromeda polifolia L. Moist site in a recently burnt-over area. Lac Brochet 3705 .

Arctostaphylos uva-ursi (L.) Spreng. Sandy beach above the shore. Cochrane R. $3689,3713$.

Arctostaphylos rubra (Rehd. \& Wils.) Fern. Muskeg site. Kasmere L. 3659 .

Loiseleuria procumbens (L.) Desv. Open black spruce forest. Kasmere L. 3656.

Oxycoccus microcarpus Turcz. Growing on Sphagnum moss in a muskeg. Kasmere L. 3653.

Vaccinium myrtilloides Michx. Recently burnt-over site. Lac Brochet 3667.

Pedicularis labradorica Wirsing Muskeg site. Kasmere L. 3654 .

Pinguicula villosa L. Growing on 
Sphagnum moss in a muskeg site. Kasmere L. 3661.

Linnaea borealis L. var. americana (Forbes) Rehd. On a wooded esker. Fort Hall L. 3735.

Viburnum edule (Michx.) Raf. Gravelly hillside. Kasmere L. 3628.

Erigeron angulosus Gaud. var. kamtschaticus (DC.) Hara Sandy shore. Fort Hall L. 3708.

Petasites sagittatus (Pursh) Gray Moist forest site. Lac Brochet 3668, 3671.

Arnica alpina (L.) Olin subsp. attenuata (Greene) Maguire North- ern slope of rocky hillside. Fort Hall. L. 3685 .

\section{LITERATURE CITED}

Baldwin, W. K. W. 1953. Botanical investigations in the Reindeer-Nueltin lakes area, Manitoba. Nat. Mus. Canada Bull. No. 128: 110-142.

Ritchie, J. C. 1959. The vegetation of northern Manitoba. III. Studies in the Subarctic. Arct. Inst. North Amer. Tech. Paper No. 3, 55 p.

Ritchie, J. C. 1962. A geobotanical survey of northern Manitoba. Arct. Inst. North Amer. Tech. Paper No. 9, 48 p.

Scoggan, H. J. 1957. Flora of Manitoba. Nat. Mus. Canada Bull. No. 140, 619 p.

\section{BALSAMROOT}

by Keith F. Best, Swift Current

Balsamroot, Balsamorhiza sagittuta (Pursh) Nutt., a tufted perennial of the sunflower family, gets both its common and scientific name from its thick, resinous (balsamlike) roots (rhiza, root) and its arrow-shaped (sagittate) leaves. Known also as Arrowleaf Balsamroot, and as Indian breadroot, this plant extends from British Columbia eastward into Saskatchewan. On many of the foothill ranges it is one of the dominant weed species, often growing in almost pure stands and commonly making up a large portion of the plant cover. It is found on well-drained soils and open, fairly dry areas such as south-facing ridges, and open sunny slopes.

Balsamroot begins growth and produces its bright yellow flowers early in the season. On native range land where plentiful, it serves as a reliable indicator of pasture readiness, as the range is generally suitable for grazing by the time that the majority of the plants are in full flower. They are valuable on spring pastures as they are fairly palatable to all classes of livestock.

Usually the seeds ripen and the leaves dry up during midsummer, but on moist sites and at higher elevations, this does not occur until late summer. Reproduction is entirely by seed which is produced in fairly large quantities. The weed is not very aggressive mainly owing to the low viability of its seed and the grazing of the flowers by livestock.
The strong, deep perennial roots enable balsamroot to withstand heavy trampling and close grazing remarkably well. These roots are often over two inches thick and exude a balsam or sticky substance with a turpentinelike odor. They have a thin corky bark and a fibrous, yellowish centre, and were used by the Indians for food.

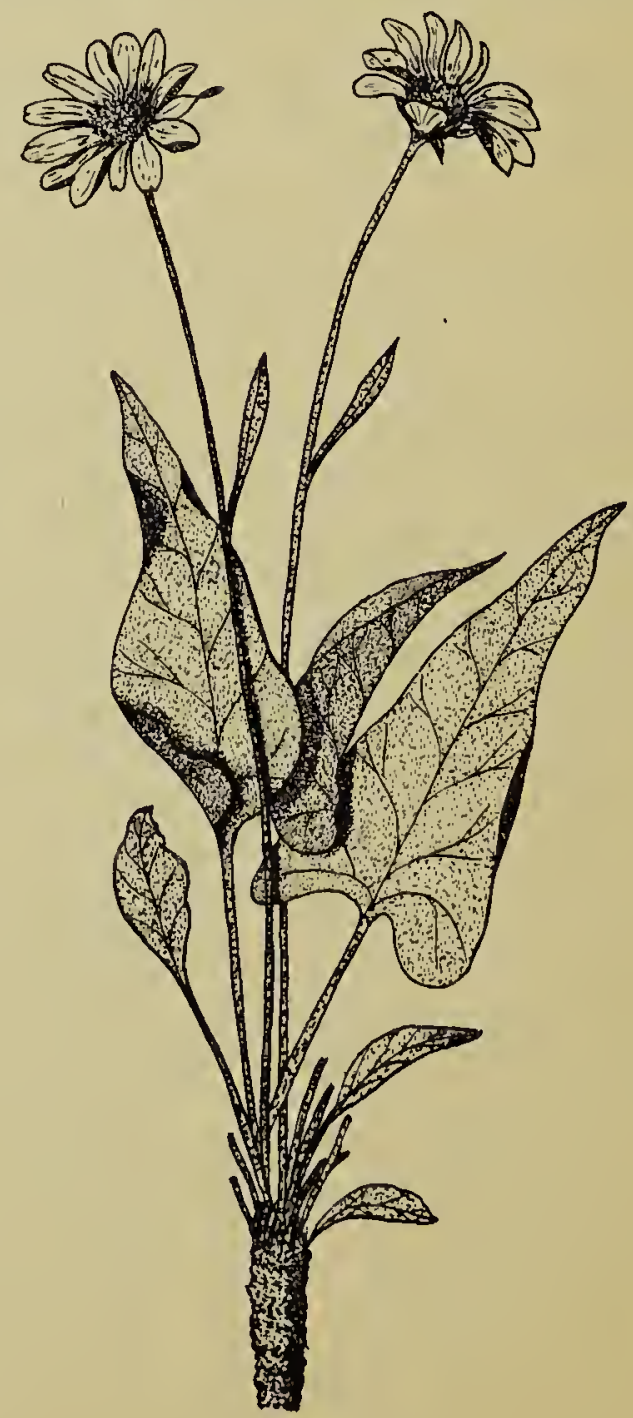

Drawing by $K$. F. Best

Balsamroot (Balsamorhiza sagittata) 